\title{
Touch-less Fingerprint Analysis - A Review and Comparison
}

\author{
Prabhjot Kaur \\ Dept. of Information Technology, \\ Maharaja Surajmal Institute of Technology, GGSIP University, New Delhi, INDIA \\ Email: thisisprabhjot@gmail.com \\ Ankit Jain \\ Dept. of Information Technology, \\ Maharaja Surajmal Institute of Technology, GGSIP University, New Delhi, INDIA \\ Email: jain.ankit66@gmail.com \\ Sonia Mittal \\ Dept. of Information Technology, \\ Maharaja Surajmal Institute of Technology, GGSIP University, New Delhi, INDIA \\ Email: soniamittal187@gmail.com
}

\begin{abstract}
Touch-less fingerprint recognition system is a reliable alternative to conventional touch-based fingerprint recognition system. Touch-less system is different from conventional system in the sense that they make use of digital camera to acquire the fingerprint image where as conventional system uses live-acquisition techniques. The conventional fingerprint systems are simple but they suffer from various problems such as hygienic, maintenance and latent fingerprints. In this paper we present a review of touch-less fingerprint recognition systems that use digital camera. We present some challenging problems that occur while developing the touch-less system. These problems are low contrast between the ridge and the valley pattern on fingerprint image, non-uniform lighting, motion blurriness and defocus, due to less depth of field of digital camera. The touch-less fingerprint recognition system can be divided into three main modules: preprocessing, feature extraction and matching. Preprocessing is an important step prior to fingerprint feature extraction and matching. In this paper we put our more emphasis on preprocessing so that the drawbacks stated earlier can be removed. Further preprocessing is divided into four parts: first is normalization, second is fingerprint Seg mentation, third is fingerprint enhancement and last is the core point detection. Feature extraction can be done by Gabor filter or by minutia extraction and the matching can be done by Support Vector Machine or Principal Component Analysis and three distance method.
\end{abstract}

Index Terms - Fingerprint, Touch-less, Seg mentation, Core-point detection, SVM (Support Vector Machine), Three Distance Method.

\section{Introduction}

Fingerprint recognition system is a biometric system that uses fingerprint as biometric input to this system. A fingerprint consists of patterns of ridges and valleys on the surface of a fingertip. Each individual has fingerprint which is different from the other. Actually this biometric system is a computer vision system which performs following functions: Image acquisition, Pre-processing, Feature Extraction, High-level processing or verification or matching. Basically, Fingerprint recognition system is an identification system that can be an Automated Fingerprint Identification System (AFIS) or a Non-automated Fingerprint Recognition System. Earlier, we used to take fingerprints using "ink techniques" in which black ink is spread on fingertip and it is pressed against a paper card, it is also called as "off-line fingerprint acquisition technique"[1]. This technique is used in the law enforcement to acquire criminal's fingerprints. Nowadays, live-scan acquisition technique is used in civil and criminal AFIS (Automated Fingerprint Identification system), that make use of sensors like optical, solid-state to acquire fingerprints.

The proposed touch-less system can be an automated system or it can be a non-automated fingerprint recognition system. The basic difference between automated and non-automated system is that the AFIS was developed by the police department for identifying persons from over a large record of files where as nonautomated fingerprint systems were developed for business purposes that replaced ID cards, passwords and other methods used for identifying. Conventional fingerprint systems are simple and require less processing as compared to the touch-less fingerprint recognition system, but they suffer from several 
problems like hygienic user interface, maintenance and latent fingerprints [2] that are invisible from naked eyes and important for law enforcement agencies.

\section{Disadvantages of Touch Based Sensors}

While using the touch-based sensor interface, the fingertip needs to be placed over the interface so that a proper fingerprint image can be taken. But the touchbased sensors have several problems like the problem of contamination which occurs because of placing the fingertip over the same interface which is already used by other. This produces a low quality fingerprint image. Another problem is due to contact pressure, which creates physical distortions which are usually non-linear in arbitrary direction and strength. Moreover, the distortion occurs globally, while its deformation parameters could be different locally in a single fingerprint image [2]. Fig 1 shows the fingerprint image of one fingertip but with different minutiae because of physical pressure [2].
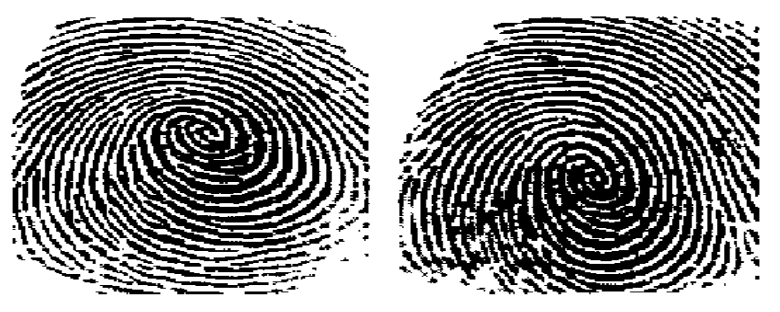

Figure 1. Physical Distortion in touch-based sensors.

So, due to the inevitable distortions of the touchbased fingerprint images, there have been attempts to make a touch less fingerprint recognition systems. Sensor, such as the ultrasound sensor, work without touch, but its large size and production cost are impractical. Moreover, they also require a long capture time, which is critical for on-line authentication systems [2]. The problem is still there and is resolved by a systemproposed in this paper.

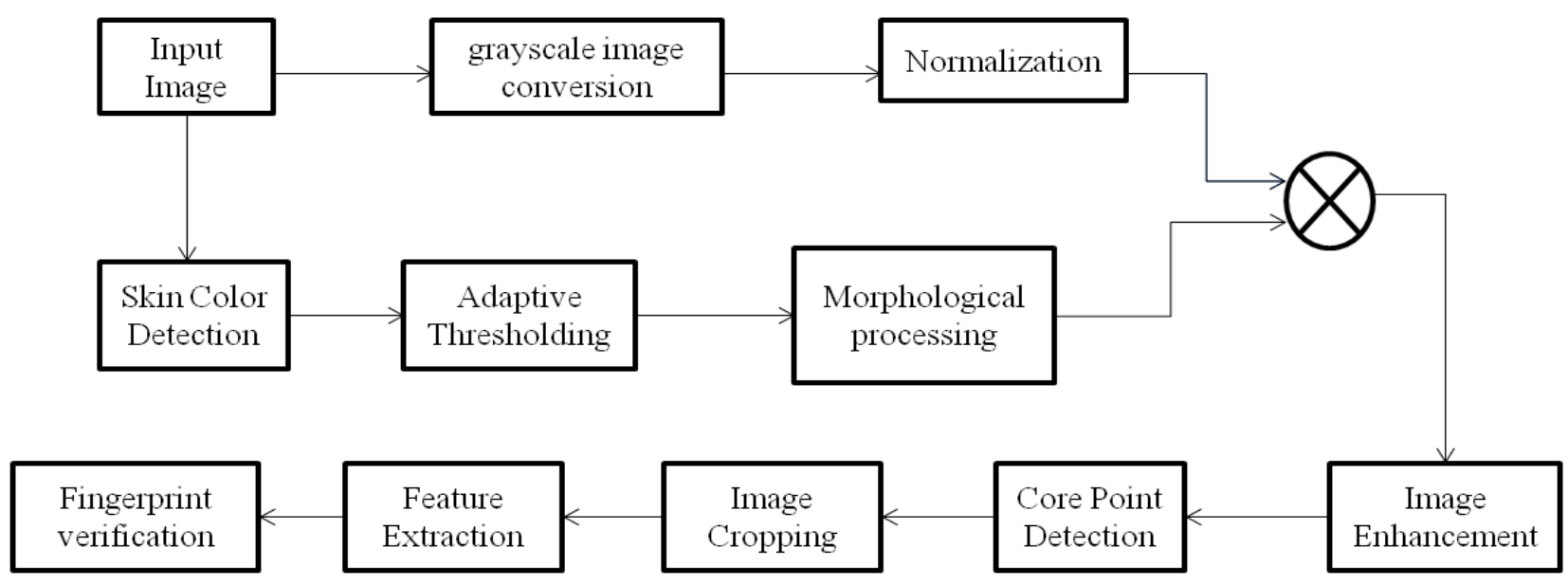

Figure 2: Block diagram of the Touch-less Fingerprint Recognition System

A touch-less fingerprint recognition system is based on remote sensing technology [3] which allows us to take fingerprint images without any physical contact as in touch-based system. The touch-less system makes use of touch-less fingerprint acquisition so as to capture the ridge-valley pattern which provides essential information for recognition [3]. Touch less fingerprint recognition systems uses digital camera to acquire the fingerprint image. There is an advantage of using digital camera i.e. the fingerprint images captured with touchless device are distortion free and present no deformation because these images are free from the pressure of contact [4].

Fingerprint images captured with touch less system are distortion free and are most desirable to acquire minutiae in the same relative location and direction at every instance because this helps the authentication system to have low FAR (false acceptance rate) and FRR (false rejection rate) [2]. While there are strong advantages of using digital camera, there appear new weak points and they are; first, the contrast between the ridges and the valleys in fingerprint images obtained with a digital camera is low. Second, the depth of the field of the camera is small, thus some part of the fingerprint regions are in focus and some parts are out of focus. Third, the problem of motion blurriness in the acquired images. Thus, the main objective is to find solutions so as to overcome these drawbacks by putting main concern on the fingerprint image preprocessing. 
The touch less fingerprint recognition system can be divided into three main modules and each module itself consists of some blocks. Each block of a module performs a special function over the input image. The three main sub-division or modules of Touch less Fingerprint Recognition System are: Pre-processing, Feature Extraction and verification or Matching. The block diagram of the touch-less fingerprint recognition systemis shown in fig. 2 .

\section{Pre-Processing}

Preprocessing is an important step prior to fingerprint feature extraction and matching. As the fingerprint images are captured using digital camera which had certain challenging problems as stated earlier so, these fingerprints require more preprocessing over them. Preprocessing is divided into four blocks.

- Normalization

- Fingerprint Segmentation

- Fingerprint Enhancement by STFT analysis

- Core Point Detection

\section{A. Normalization}

Normalization is the first preprocessing operation. It can be done in two ways for two different purposes. In the first way [3], normalization is done so as to minimize the non-uniform lighting problem. It can be done by changing the dynamic range of the pixel intensity values. It calculates the mean and variance of an image and thus reduces the difference of the illumination. The normalization of $f(x, y)$ is computed as follows:

$g(x, y)=\frac{f(x, y)-m_{x}(x, y)}{\sigma_{f}(x, y)}$

Where

$f(x, y)$ : The original image

$m_{f}(x, y):$ An estimation of a mean $f(x, y)$

$\sigma_{f}(x, y):$ An estimation of the standard deviation

The estimation of the mean and standard deviation is performed through spatial s moothing. The other way, an input fingerprint image is normalized so that it has a pre-specified mean and variance [5]. Normalization of an image is carried out after conversion of image from RGB to grayscale. The fingerprint images captured by using digital camera are in RGB format, so RGB to grayscale image conversion is done. A grayscale image has 256 different gray levels which are sufficient for the recognition of most natural objects .In a gray-level fingerprint image, ridges and valleys in a local neighborhood form a sinusoidal-shaped plane wave which has a well-defined frequency and orientation. Now after conversion, the grayscale image is then given as an input to the normalization block where the image can be normalized. Let $I(i, j)$ denote the gray-level value at pixel $(i, j), M$ and $V$ denote the estimated mean and variance of $I$, respectively, and $G(i, j)$ denote the normalized gray-level value at pixel $(i, j)$. The normalized image is defined as follows:

$= \begin{cases}M_{o}+\sqrt{\frac{V_{o}(I(i, j)-M)^{2}}{V}} & \text { if } I(i, j)>M \\ M_{o}-\sqrt{\frac{V_{o}(I(i, j)-M)^{2}}{V}} & \text { otherwise }\end{cases}$

Where $M_{o}$ and $V_{o}$ are the desired mean and variance values, respectively. Normalization is a pixel-wise operation. It does not change the clarity of the ridge and valley structures.

\section{B. Fingerprint Segmentation}

In parallel with the normalization process fingerprint segmentation is also going on. Segmentation of fingerprint image is necessary so as to reduce the size of the input data, to eliminate undesired background [4], which is the noisy and to focus on area which is in favor of the central part of the fingerprint. The proposed fingerprint segmentation is further divided into three processes: skin color detection, adaptive thresholding, morphological processing. The algorithm proposed for skin color detection [4] is:

1. The image is converted from RGB color space to $\mathrm{YCbCr}$ color space. Segmentation of the skin color regions becomes robust if only the chrominance component is used in analysis. Therefore, the variations of lu minance component are eliminated by choosing the $\mathrm{CbCr}$ plane (chrominance components) of the $\mathrm{YCbCr}$ color space to determine the probable fingerprint region. The brightness component contained in the $\mathrm{Y}$ of the YCbCr will not be used.

2. Then, those images are low pass filtered to remove noise. The filter mask for the low pass filtering is as below and it can be said as "average filtering".

$$
1 / 9\left[\begin{array}{lll}
1 & 1 & 1 \\
1 & 1 & 1 \\
1 & 1 & 1
\end{array}\right]
$$

3. Mean and covariance for $\mathrm{Cb}$ and $\mathrm{Cr}$ are determined by:

Mean: $m=E(x) \quad$ where $x=\left[\begin{array}{ll}C b & C r\end{array}\right]^{T}$

Covariance $\quad C=E\left[(x-m)(x-m)^{T}\right]$

4. Region of interest is determined by using:

$P\left(\frac{x}{\text { skin }}\right)=\exp \left\lfloor-0.5(x-m)^{T} C^{-1}(x-m)\right\rfloor$ 
After the region of interest is determined, the image is thresholded into a binary image with adaptive thresholding. If the background has close resemblance to the skin color, it will be taken as fingerprint. To solve this problem, binary image processing technique is applied. The purpose of this process is to smooth, fill in, and/or remove objects in a binary image. Now, the binary mask from segmentation is multiplied with the normalized image.

\section{Fingerprint Enhancement using STFT Analysis}

The fingerprint image may be thought of as a system of oriented texture with the local ridge orientation and ridge frequency varying slowly throughout the image [6]. Due to this non-stationary nature of the image, traditional Fourier analysis is not adequate to analyze the image completely. So it is required to resolve the properties of the image both in space and also in frequency [6]. The steps in this proposed analysis [6] are:

The image is first divided into overlapping windows during STFT analysis. The overlapping window is used to preserve the ridge continuity and removes 'block' effects common with other block processing image operations. The image is assumed stationary within this small window and can be modeled approximately as a surface wave. Probabilistic estimates of the ridge frequency, ridge orientation and energy map are obtained after the Fourier spectrum of this small region is analyzed

The Fourier spectrum is represented in polar form $\operatorname{as} F(r, \theta)$. A probability density function $p(r, \theta)$ and the marginal density functions $p(\theta), p(r)$ are defined as

$$
\begin{aligned}
& p(r, \theta)=\frac{|F(r, \theta)|^{2}}{\int_{r} \int_{\theta}|F(r, \theta)|^{2}} \\
& p(r)=\int_{\theta} p(r, \theta) d \theta, \quad p(\theta)=\int_{r} p(r, \theta) d r
\end{aligned}
$$

\section{(i) Ridge Orientation Image}

Assume that the orientation $\theta$ is a random variable that has the probability density function $p(\theta)$. The expected value of the orientation may then be obtained by performing a vector averaging.

$$
\begin{aligned}
& E\{\theta\} \\
& =\frac{1}{2} \tan ^{-1}\left\{\frac{\int_{\theta} p(\theta) \sin (2 \theta) d \theta}{\int_{\theta} p(\theta) \cos (2 \theta) d \theta}\right\}
\end{aligned}
$$

The resulted orientation image $O(x, y)$ obtained from above equation is further smoothened using vectorial averaging and the smoothened image $O^{\prime}(x, y)$ is obtained.

\section{(ii) Ridge Frequency Image}

The average ridge frequency is estimated in a manner similar to the ridge orientation. Assume the ridge frequency to be a random variable with the probability density function $p(r)$. The expected value of the ridge frequency is given by the following:

$$
\begin{aligned}
& E(r) \\
& =\int_{r} p(r) r d r
\end{aligned}
$$

The frequency map so obtained is smoothened using a $3 \times 3$ Gaussian mask.

\section{(iii) Region Mask}

Simply, energy map is used as a region mask to get finer fingerprint segmentation. The region mask is obtained by using Otsu's optimal thresholding technique. The energy map is obtained by:

$$
\begin{aligned}
& E(x, y) \\
& =\log \left\{\int_{r} \int_{\theta}|F(r, \theta)|^{2}\right\}
\end{aligned}
$$

The orientation image is then used in computing coherence image to prevent spurious artifacts caused by the discontinuities in the ridge flow at the block boundary especially at in the regions of high curvature close to the core and deltas which have more than one dominant orientation.

\section{Core point Detection}

To differentiate the entries of fingerprint images singular points, SPs are used. SPs are points that can be consistently detected in a fingerprint image and can be used as a registration point. Typically there are two types of singular points: core point and delta point. In this paper we only proposed the core point detection method. Fingerprint's core point can be defined as the point of maximum curvature in the fingerprint image.

A fingerprint can have two structures, the global and the local structure. In the global structure the overall pattern of the ridges and valleys are considered where as in local structure the detailed pattern around a minutiae point is considered. A minutiae point is a position in the fingerprint where a ridge is suddenly broken or two ridges are merged.

The global structure is used because it is more stable even when the fingerprint is of poor quality. Core points have special symmetry properties which make them easy to identify also by humans. 
Core Point detection can be done by using complex filtering [10]. The algorithm proposed for core point detection is:

1. Complex filter of order $m$ are modeled by $\exp \{i m \varphi\}$. A polynomial approximation of these filters in Gaussian windows yield $(x+$ iy) ${ }^{m} g(x, y)$ where $g$ is a Gaussian defined as $g(x, y)=$ $\exp \left\{-\frac{x^{2}+y^{2}}{2 \sigma^{2}}\right\}$.

2. Now these filters are applied not directly to the original enhanced fingerprint image but they are applied to the complex valued orientation tensor field image $z(x, y)=\left(f_{x}+i f_{y}\right)^{2}$ where $f_{x}$ is the derivative of the original image in the x-direction and $f_{y}$ is the derivative in the y-direction.

3. Filters of first order symmetry are used i.e.

For core Point:

$$
\begin{aligned}
& h_{1}(x, y)=(x+i y) g(x, y) \\
& =r \exp \{i \varphi\} g(x, y)
\end{aligned}
$$

For delta point:

$$
h_{2}(x, y)=(x-i y) g(x, y)=r \exp \{-i \varphi\} g(x, y)
$$

4. The complex filter response is $c=\mu \exp \{i \alpha\}$, where $\mu$ is a measure of symmetry and $\alpha$ is the "member" of that symmetry family. By using certainty measures $\mu_{1}$ and $\mu_{2}$ for core point and delta point respectively, we can identify an SP of type core if $\left|\mu_{1}\right|>T_{1}$ and of type delta if $\left|\mu_{2}\right|>T_{2}$, where $T_{1}$ and $T_{2}$ are threshold.

5. Then, multi-scale filtering is used to extract SPs more robustly and precisely compared to a representation at only one resolution level. The extraction of an SP starts at the lowest resolution level and continues with refinement at higher resolutions.

6. The complex orientation field $z(x, y)$ is represented by a four level gaussian pyramid. Level 3 has the lowest, and level 0 has the highest resolution. The core and the delta filtering are applied on each resolution. The complex filter response is called $c_{n k}$ where $\mathrm{k}=3,2,1$ and 0 are the resolution levels, and $n=1,2$ are the filter types.

7. In order to improve the selectivity of the filters, i.e. a filter should give a strong response only to one of the symmetries so we have used the following rules to sharpen the magnitude of the filter responses:

$$
\left\{\begin{array}{l}
s_{1 k}=\mu_{1 k}\left(1-\mu_{2 k}\right) \\
s_{2 k}=\mu_{2 k}\left(1-\mu_{1 k}\right)
\end{array}\right.
$$

With levels $\mathrm{k}=0,1,2$, and 3. The complex filter response is $c_{n k}=s_{n k} \exp \left\{i \alpha_{n k}\right\}$ where $s_{n k}$ is a measure of certainty for that there is a symmetry of type $n$ at resolution $k$, and $\alpha_{n k}$ is how much the symmetric pattern is rotated to a fixed reference.

After the core point is detected the enhanced image is cropped to size $200 \times 200$ in which the core point is at the center of the image.

\section{Feature Extraction}

Most fingerprint identification methods use minutiae as the fingerprint features [7]. The steps involved in minutiae extraction are smoothing, local ridge orientation estimation, ridge extraction, and thinning and minutiae detection. But for a s mall scale system, it is not efficient to process all the steps. So, we proposed Gabor filter-based feature extractor [8] because its frequency and orientation representation are similar to those of human visual system. Also Gabor filter helps in smoothing out noise and preserving true ridge valley structures.

General form of 2D Gabor filter is:

$h\left(x, y, \theta_{k}, f, \sigma_{x}, \sigma_{y}\right)=\exp \left[-\frac{1}{2}\left(\frac{x_{\theta_{k}}^{2}}{\sigma_{x}^{2}}+\frac{y_{\theta_{k}}^{2}}{\sigma_{y}^{2}}\right)\right]$

$\times \exp \left(i 2 \pi f x \theta_{k}\right)$

where

$x_{\theta_{k}}=x \cos \theta_{k}+y \sin \theta_{k}$,

$y_{\theta_{k}}=-x \sin \theta_{k}+y \cos \theta_{k}$,

$f$ is the frequency of the sinusoidal plane wave,

$\theta_{k}$ is the orientation of the Gabor Filter,

$\sigma_{x}$ and $\sigma_{y}$ are the standard deviations of the Gaussian envelope along the $\mathrm{x}$ and $\mathrm{y}$ axes respectively.

When core point is detected, the cropped normalize image is cropped again into size of $200 \times 200$ taking core point as center. Then images are sampled using Gabor filters and afterwards these filtered images are divided into set of $8 \times 8$ non-overlapping blocks. After that standard deviation is calculated which results in a scalar nu mber and it forms Gabor feature of each image. All features collected are scaled [3] to range of $[0,1]$ by

$$
n=(s-m) /(M-m)
$$

where

$\mathrm{n}$ : normalized feature.

$\mathrm{s}$ : original feature.

M: maximum value of all features.

$\mathrm{m}$ : minimum value of all features. 


\section{Fingerprint Verification}

Fingerprint verification is done by SVM [3]. SVM is a binary classifier which is based on the principle of structural risk minimization and maps an input sample to a high-dimensional feature space [10]. SVM could optimally separate the two classes of genuine and imposters by constructing a hyper-plane. The training dataset is labeled as

$\left\{x_{i}, y_{i}\right\}, x_{i} \in \mathfrak{R}^{d}, y_{i} \in\{+1,-1\}, i=1 \ldots \ldots N$

Where $x_{i}$ are Gabor features extracted belongs either to genuine or imposter class, $y_{i}$ is class label $(+1$ for genuine, -1 for imposter). Suppose we have a hyperplane that distinguish positive from negative examples. It is formulated as

$$
\begin{aligned}
& x \cdot w+b \\
& =0
\end{aligned}
$$

where:

$\mathrm{W}$ is normal to plane.

$\mathrm{b}$ is the bias term.

For finding hyper-plane which separate two classes which has maximum distance to closest point on each side of that plane, square of L2-norm of $\mathrm{w}$ where $\|\mathrm{w}\|_{2}^{2}$ is imposed to inequalities $\left(\mathrm{x}_{i} \cdot \mathrm{w}+\mathrm{b}=0\right) y_{i}>=1$ for all $\mathrm{I}$ is minimized.

Each data point is transformed to a higher dimensional space for extending to non-linear boundaries. Proper transformation is required for achieving better separatibility between the two classes. For performing this transformation polynomial kernel function as formulated in (18) or radial basis function kernel as formulated in (19) are used.

$$
K\left(x_{i}, x_{j}\right)=\left(x_{i} \cdot x_{j}+1\right)^{n}
$$

where $\mathrm{n}$ is order of polynomial.

$K\left(x_{i}, x_{j}\right)=\exp \left[-\frac{1}{2}\left(\frac{|| x_{i}-x_{j}||}{\sigma}\right)^{2}\right]$

where $\sigma$ is width of radial basis function.

Another Method for verification is PCA (Principal Component Analysis) and Three Distance Measures [11]. Since the number of Gabor features extracted is huge, PCA is used for decreasing dimensionality of the feature vectors and retaining only those characteristics of feature vectors which contributing most its variance and eliminate later principal components. For matching three distance measures are used which are Manhattan distance, Euclidean distance and Cosine angle.
The Manhattan distance $\left(\mathrm{L}_{1}\right.$-norm) is addition of absolute difference between two feature vectors. It is formulated as follows:

$d_{L 1}(y, x)=\sum_{i=1}^{n}\left|x_{i}-y_{i}\right|$

The Euclidean distance $\left(\mathrm{L}_{2}\right.$-norm) is the distance between feature vectors derived as a straight line and most commonly used calculation.

$d_{L 2}(y, x)$
$=\sqrt{\sum_{i=1}^{n}\left|x_{i}-y_{i}\right|^{2}}$

The Cosine angle is cosine of angle between two feature vectors. It is calculated as:

$$
\begin{aligned}
& d_{\cos (y, x)}(y \cdot x \\
& =\frac{|y||x|}{}
\end{aligned}
$$

Let $d$ is the distance computed fromeq. (20), (21) and (22), and threshold is set to T, matching is done if $\mathrm{d}<\mathrm{T}$ and rejected when $\mathrm{d} \geq \mathrm{T}$.

\section{Conclusion}

In this paper, a review of touch-less fingerprint recognition system, which can be an automated or a biometric digital camera based system is presented. We also presented number of comparisons between touchless systems and the conventional fingerprint recognition systems. Further, this paper presented a modeled system that comprised of preprocessing, feature extraction and matching. Preprocessing is further subdivided into normalization, segmentation, enhancement and core point detection. The feature extraction might be based on minutiae extraction or image based method that is Gabor filter in which feature vectors are extracted. Moreover we have presented an effective verification technique that employs the SVM classifier and compares it with three distance measures.

\section{Future Scope}

We are looking forward for discovering a better enhancement method for fingerprint images captured by the digital camera by introducing some concepts of soft computing so that the output of preprocessing module increases the performance of system. We are also trying to discover more optimal methods for matching the fingerprint images. We are also directed towards removing the limitation due to digital camera and also to make this system to work with mobile phone so that fingerprint images can be captured by it 


\section{References}

[1] D. Maltoni, D. Maio, A. K.Jain, and S. Prabhakar, Handbook of Fingerprint Recognition, SpringerVerlag, New York, 2003.

[2] Y. Song, C. Lee, and J. Kim, "A New Scheme for Touchless Fingerprint Recognition System", International Symposium on Intelligent Signal Processing and Communication Systems, Korea, 2004.

[3] B.Y. Hiew, Andrew B.J. Teoh and Y.H. Pang, "Touch-less fingerprint recognition system", 2007.

[4] B.Y. Hiew, Andrew B.J. Teoh, and David C.L. Ngo, "Preprocessing of Fingerprint Images Captured with a Digital Camera", 9th International Conference on Control, Automation, Robotics and Vision (ICARCV 2006), Singapore, 2006.

[5] Lin Hong, Student Member, IEEE, Yifei Wan, and Anil Jain "Fingerprint Image Enhancement: Algorithm and Performance Evaluation", IEEE Transactions on Pattern Analysis and Machine Intelligence, Vol. 20, No. 8, August 1998.

[6] S. Chikkerur, A. Cartwright, and V. Govindaraju, "Fingerprint Image Enhancement Using STFT Analysis", International Conference on Advances in Pattern Recognition, United Kingdom, 2005.

[7] Jain, A., Hong, L., and Bolle, R.:"On-line Fingerprint Verification", IEEE Transaction, 1997.

[8] C.J. Lee and S.D. Wang, "Fingerprint feature extraction using Gabor filters", Electronics Letters, vol.35, no.4, 1999.

[9] Kenneth Nilss on and Josef Bigun, "Localization of corresponding points in fingerprints by complex filtering” ,Pattern Recognition Letters, vol. 24, pp. 2135-2144, 2003.

[10] Burges, C.J.C., "A tutorial on Support vector Machine for Pattern Recognition", Knowledge Discovery and Data Mining, vol.2, no.2, 1998.

[11] B.Y. Hiew, Andrew B.J. Teoh and Y.H. Pang, "Digital camera based fingerprint recognition", 2007 IEEE international conference on Telecommunications.

Prabhjot Kaur has completed her B. Tech. in 1999 and M. Tech. in 2003. Presently she is working as a Reader in Maharaja Surajmal Institute of Technology and pursuing her P.hD. from Sharda University, Greater Noida, New Delhi.

Ankit Jain is pursuing his Bachelor of Information Technology from Maharaja Surajmal Institute of Technology, GGSIPU, New Delhi. Currently, he is doing his major project on Implementation of Touch- less Fingerprint Recognition System. His research interests are biometrics, image processing and fingerprint systems.

Sonia Mittal is pursuing her Bachelor of Information Technology from Maharaja Surajmal Institute of Technology, GGSIPU, New Delhi. Currently, she is doing her major project on Implementation of Touchless Fingerprint Recognition System. Her areas of interest are soft computing and image processing.

How to cite this paper: Prabhjot Kaur,Ankit Jain,Sonia Mittal,"Touch-less Fingerprint Analysis - A Review and Comparison", International Journal of Intelligent Systems and Applications(IJISA), vol.4, no.6, pp.46-52, 2012. DOI: 10.5815/ijisa.2012.06.06 